\title{
ENERGY EFFICIENT MULTI-EVENT ROUTING IN WSN
}

\author{
Ayan Kumar Das ${ }^{1}$ And Rituparna Chaki ${ }^{2}$ \\ ${ }^{1}$ Department of Information Technology, Calcutta Institute of Engineering and \\ Management, Kolkata, India \\ ayandas24114057@yahoo.co.in \\ ${ }^{2}$ Department of Computer Science and Engineering, West Bengal University of \\ Technology, Kolkata, India \\ rituchaki@gmail.com
}

\begin{abstract}
Researchers have come up with various routing solutions for WSNs. Some of the algorithms concentrate on setting up an event path to send that information; some other algorithms flood that information throughout the network. These techniques suffer from extra power requirement, making them unsatisfactory in case of WSNs. This paper proposes to handle multiple events routing in an energy efficient manner. The technique also takes care of congestion in the network and manages the problem of infinite loop.
\end{abstract}

\section{KEYWORDS}

Event categorization, Congestion Control, Event Priority, Event Number, Network longevity, Flooding, Dead End and Infinite loop.

\section{INTRODUCTION}

A Sensor node is a small battery operated mobile device and capable of computation and communication. A Wireless Sensor Network (WSN) consists of spatially distributed autonomous sensors to monitor physical or environmental conditions, such as temperature, sound, vibration, pressure, motion or pollutants etc. The sensed information is then cooperatively passed through the network to a single point called the sink node. The WSNs, like MANETs, essentially support dynamic network topology. Their ability to withstand difficult environmental conditions, coupled with ease of deployment and high scalability had made them a popular choice with the military and disaster management groups in the past. Wireless sensor networks facilitate monitoring and controlling of physical environments from remote locations with better accuracy. Therefore, the quantity and quality of the data delivered to the end-user is very important. The sensors are essentially very small in size, thus limiting their battery and storage capacities. The nature of deployment of sensor nodes, makes battery recharge nearly impossible. The battery power of a highly active sensor node thus gets exhausted very fast. This often causes a part of the WSN involved in crucial event sensing, to get disconnected. In disaster management and natural calamity forecasting, often this hampers the flow of vital information. The seriousness of damages caused due to the quick battery drainage makes power management the most challenging issues of WSN.

The current state of the art study shows that most of the power saving algorithms highly concentrates on sending signals to a base station through an event path. Again some other DOI: $10.5121 /$ ijassn.2012.2402 
algorithms flood the information about the event from its source node to neighbour nodes and those nodes again flood their neighbours. In both the above cases these algorithms are capable of sending information's to the base station successfully but their major drawbacks are-

In the first case the nodes forming the event path deplete their energy more quickly than the other nodes as the event path is a fixed path to propagate the signal from source node to base station. This may cause an unstable uniform power of the whole system leading to system failure. The query nodes may get the event information in very late if it sends its query irregularly. Otherwise, if the query node sends the query regularly then the power consumption will be very high for that node.

In the second case, the source node floods information of devastating event and the respective neighbour nodes also flood the received information, which leads to high power drainage for all the nodes. Flooding also cause to redundancy in packet movement, leading to route congestion and eventual power loss.

The proposed algorithm is designed to handle multiple events and to reduce the congestion of the network by balancing the power of the overall network, so that the longevity of the network may increase. Apart from that the algorithm also solves the infinite loop problem and concern about data loss also. In this algorithm every node finds the shortest path to reach the base station. Here events should be categorized as Higher Priority Event (i.e. the node has sensed the devastating data) and Lower Priority Event (i.e. the node has sensed non devastating data). If Higher Priority Event occurs then the source node will send its information along with shortest path, otherwise it will send to the neighbour node, which is previously not visited and has maximum power and it will send its neighbour and so on. Here every node has the node numbers of the previously visited nodes, so that if the data packets reach at the dead end then is will be able to come back and find its destination again. Thus in case of devastating situation the data packet will reach the base station in shortest path and it will take minimum time, when for non devastating situation the data packet will be sent by the nodes which has the maximum power. Thus the algorithm maintains the overall energy of the network at the same time the base station gets the devastating information in very short time.

The remaining part of this paper is organized as follows: Section 2 deals with the review of state of the art routing topologies, section 3 gives a description of the proposed methodology, section 4 contains the simulation reports and section 5 is the concluding part.

\section{BACKGROUND}

The following section gives a glimpse of the research work being undertaken in this field.

\subsection{Power Aware Routing Algorithms}

Standard In this type of routing the main technique is focused on the effect of power efficient routing on the lifetime of multi hop wireless sensor networks (WSNs). All the nodes in the WSNs are divided into tiers. The nodes belonging to the highest tier are very important, because these nodes imply higher power consumption than that of nodes of any other tier. Obviously the batteries of nodes of $1^{\text {st }}$ tier depletes sooner than these of any other tier. As soon as the nodes of $1^{\text {st }}$ tier dies, the network becomes disconnected.

Energy Efficient Routing for Single Destination Flow redirection algorithm (FR) [5] is that type of algorithm in which we have a Largest longest length path (Lp) which is the path with largest capacity in terms of battery power and has less energy consumption per bit transmission than all 
other nodes in network and Smallest longest length path $(\mathrm{Sp})$ which is the path with minimum capacity in terms of battery power and have higher energy consumption per bit transmission than all other nodes in network. It is the redirection based algorithm where some amount of flow is redirected from smallest longest length path (Sp) to largest longest length path (Lp). This algorithm firstly determines the two paths from source node to the destination which are to be involved in redirection. After that it calculates the amount of redirection $(€)$ and then $€$ amount of flow will be added to the largest longest length path Lp. After adding the algorithm checks one or possibly more loops in the path and then loops are removed link by link along the path so that the flows of the two paths can be properly incremented or decremented.

Maximum Residual Energy Path Routing (MREP) [11] algorithm is designed to augment the flow on the path whose minimum residual energy after the flow augmentation will be longest. This algorithm assumes that the topology of the network is static. Hence the results are applicable to networks which are either static like sensor network or whose topology changes slowly enough, but not applicable for ad hoc network whose topology changes very frequently. The algorithm used fixed information generation rates and required a priori knowledge of future information generation.

A Novel Power-Balancing Routing Scheme for WSN [3] is designed to detect the source of the network. In this algorithm the source node floods the event information along an event path and the other nodes send the queries at a regular time interval. A node sends the query to its neighbour consisting of maximum power and then the neighbour of neighbour and so on. When the query finds a node of event path then it detects the source node along with event path. The algorithm also guarantees that the query packet will not get an infinite loop by status checking mechanism and thus increasing the longevity of the overall network.

Intelligent Energy Efficient Routing for Ad-Hoc Sensor Network by Designing QCS Protocol [4] states that depending on the type of information generated on a particular node or signal transmitted from a particular node to other node can be of three types- a) Regular information, b)Irregular Information, c)Devastating - Immediate Response Information. Here the network goes through mainly three stages - Nodes placing, Node activation and Node communication. In "QCS protocol" which stands for "QUERY-CHECKED-SOURCE" protocol, all the nodes of the network are of same type but all of them can behave in three different categories - QUERY NODE, CHECKED NODE and SOURCE NODE, depending on the situation. Signals are categorized into three types, 1.regular, 2.irregular \& 3.devastating depending upon the sensed value by the sensor. Network Initialization is done in the following way- A node is activated as "Q" node (Query node) randomly from all nodes in the network. The one non adjacent node of "Q" is chosen and denoted as "Q". This procedure continues until all the nodes nonadjacent to each other are chosen and denoted as "Q". Query node will query first and those nodes which will answer be the "C" nodes, i.e. checked nodes. At the next moment (after the time period " $T$ ") " $C$ " nodes turn into "Q" and will repeat the procedure. The above procedure is true for type 1 \&type 2 signals. The type 3 signal will be flooded following Final Broad Cast-Petrol Flow protocol only, as it is an emergency \& make all adjacent as " $\mathrm{S}$ " and continue.

Load-Balanced Minimum Energy Routing (LBMER) [6] algorithm maximizes the network lifetime, as the energy-constraint sensor nodes in WSNs operate on limited batteries, it is a very important issue to use energy efficiently and reduce power consumption and for that it is essential to prolong each individual node's lifetime by minimizing the transmission energy consumption. This algorithm for WSNs considers both sensor node's energy consumption status and the sensor node's hierarchical congestion levels. It uses mixture of energy balance and traffic balance to solve the problem of congestion of WSNs and avoid the situation of congestion in sensor nodes using their energy at much higher rate and die much faster than the other nodes. The path for routing established by LBMER will not be very congested and the traffic will be distributed 
evenly in the WSNs. Simulation results verified that the LBMER performance is better than that of Min-Hop routing and the existing minimum energy routing scheme MTPR.

\subsection{Cluster Based Routing Algorithm}

The In clustered network, nodes are clustered in the form of hierarchical structure. The advantage of cluster based method is improving routing efficiency, scalability, supporting QOS and saving power consumption in the nodes. Generally clustering transforms a physical network into a virtual network which has interconnected clusters.

Low Energy Adaptive Clustering Hierarchy (LEACH) [12] is also cluster-based protocol, which includes distributed cluster formation. LEACH randomly selects a few sensor nodes as cluster heads $(\mathrm{CHs})$ and rotates this role to evenly distribute the energy load among the sensors in the network. In LEACH, the cluster head $(\mathrm{CH})$ nodes compress data arriving from nodes that belong to the respective cluster, and send an aggregated packet to the base station in order to reduce the amount of information that must be transmitted to the base station. LEACH uses a TDMA/CDMA MAC to reduce inter-cluster and intra-cluster collisions. However, data collection is centralized and is performed periodically. Therefore, this protocol is most appropriate when there is a need for constant monitoring by the sensor network. A user may not need all the data immediately. Hence, periodic data transmissions are unnecessary which may drain the limited energy of the sensor nodes. After a given interval of time, a randomized rotation of the role of the $\mathrm{CH}$ is conducted so that uniform energy dissipation in the sensor network is obtained. The authors showed that only $5 \%$ of the nodes need to act as cluster heads.

Hybrid Energy-Efficient Distributed (HEED) routing [14] is also a clustering approach, which is one of the most recognized energy-efficient clustering protocols. It extends the basic scheme of LEACH by using residual energy and node degree or density. In HEED, the initial probability for each node to become a tentative cluster head depends on its residual energy, and final heads are selected according to the intra-cluster communication cost. The clustering process is divided into a number of iterations, and terminates with in a constant number of iterations. HEED achieves fairly uniform distribution of cluster heads across the network.

\subsection{Agent Based Routing}

Author Energy-Efficient Ant-Based Routing algorithm (EEABR) [8] is based on the Ant Colony Optimization (ACO). EEABR uses a colony of artificial ants that travel through the WSN looking for paths between the sensor nodes and a destination node, that are at the same time short in length and energy-efficient, contributing in that way to maximize the lifetime of the WSN. Each ant chooses the next node to go with a probability that is a function of the energy of the nodes. When an ant reaches the destination node, it travels backwards trough the path constructed and updates the pheromone trail by an amount that is based on the energy quality and the number of nodes of the path. After some iteration the EEABR protocol is able to build a routing tree with optimized energy branches.

Ant-Colony Based Routing Algorithm (ARA) [15] used distance vector routing. Route discovery in ARA is done by broadcasting Forward Ants (FANT). ARA implements the packet sequencing mechanism to prevent packet loops. In ARA, destinations respond to multiple FANT packets received from the same node, thus supporting multi path routing. 


\subsection{Query Driven Routing}

In Information-Driven Sensor Querying (IDSQ) [17], the querying node is capable of determining which node can provide the useful information with the advantage of balancing the energy cost. However, IDSQ does not specifically define how the query and the information are routed between sensors and the base station. Therefore, IDSQ can be seen as a complementary optimization procedure. Simulation shows that these approaches are more energy-efficient than directed diffusion where queries are diffused in an isotropic fashion and reaching nearest neighbours first.

\subsection{Zone Based Routing}

The zone-based routing algorithm [2] generally follows a method in which max-min coordinate system is used. It is used for large scale networks and is fairly good to optimize the lifetime of the network. Zone-base routing is a hierarchical method where the area covered by the sensor network is divided into a small number of zones. Each zone has many nodes and thus a lot of redundancy occurs in routing a message through it. To send a message across the entire area it finds a global path from zone to zone and gives each zone control over how to route the message within itself. A local path for the message is computes within each zone so as to not decrease the power level of the zone too much.

\section{Proposed Work}

\subsection{Basic Concept}

Optimization of power consumption within the entire network is one of the main challenges in WSN. The information about the devastating event should be sent to the base station by shortest path, as the base station should know about the devastating information as soon as possible. This may lead to high power consumption of the nodes belong to that shortest path, which may cause breaking down of that path. This may be prevented by sending the information along with the path which has the maximum power. Obviously the second one will take more time than by sending the information with the shortest path. Thus event categorization can ease the above problem by giving high or low priority to the events depending on their destruction ability, where high priority events will be sent by shortest path and low priority events will be sent by the path which has the maximum power.

Infinite loop problem can be considered as another challenge of the above type of routing in WSN. A node will send information of low priority event to its neighbour node which has the maximum power and then that neighbour will send it to neighbour of neighbour containing maximum power and so on. This process may cause two problems; firstly the data packet is sent back to its source again and will create an infinite loop, which should be avoided. Secondly it may send the data packet to a dead end instead of base station that is there may be data loss.

Congestion is another reason of delay in the network. This amount of time should be reduced as the base station should know the information about the events as early as possible, so that it can take the corrective action.

Thus information about the events should reach the base station in very short time and less energy consumption should also be coupled with this for the shake of longevity of the overall network. Keeping in mind these factors, the proposed algorithm introduces - event categorization, solution of infinite loop problem, preventing data loss, multiple event handling and congestion control. 
The Event Categorization is done to set priority to events that has occurred in the vicinity of the nodes. For example, if an earthquake occurs and its intensity is computed by the node in the range of 7 and above on the Richter scale then treats that as a higher priority event. When the intensity is below 7 in Richter scale, then the event is treated as a lower priority event. The data packets with higher priority event will be sent through the shortest path from source node to base station so that it can take very less time and we are not concerning about the power at that time. On the other hand, in case of lower priority event data packets should be sent by searching the neighbour nodes consisting of maximum power, after checking that the node is not previously visited by status checking mechanism to solve the infinite loop problem. The header of the data packet is maintaining the node numbers so that if it reaches to the dead end then it will be able to come back by previous path and find the base station. The proposed algorithm aims to send disaster related information very fast so that the base station can take necessary action and send non devastating information by checking the power of neighbour nodes so that the longevity of the overall network may increase.

At a time multiple events may occur in different nodes. Then it will have different source nodes. Every source node will start sending data packets of different information of different priority. Now it may happen that for one node multiple data packets are coming from different source nodes and sending all the packets at a time from this node may cause the congestion of the network.

To control the congestion of the network the algorithm is maintaining an event queue, which can receive 2 high priority packets and 4 low priority packets, when it can transmit only one packet at a time interval of every $2 \mathrm{~ms}$. The queue will transmit the high priority packet first, when the packets of same priority will be sent on first come first serve basis.

\subsection{Data Dictionary}

Table 1. Variables list

\begin{tabular}{|c|c|}
\hline Variable Name & Description \\
\hline $\mathrm{N}$ & Total Number of nodes \\
\hline $\mathrm{P}$ & Initial power for all the nodes \\
\hline Ep & Event priority value \\
\hline $\operatorname{arr}[]$ & $\begin{array}{l}\text { An array consists of the connections } \\
\text { between the nodes }\end{array}$ \\
\hline s_path[] & $\begin{array}{l}\text { List of all the nodes for shortest path from } \\
\text { a source node }\end{array}$ \\
\hline power[node_id][energy] & $\begin{array}{l}\text { An array consists of initial power of each } \\
\text { node }\end{array}$ \\
\hline status[node_id] & Visited nodes are stored in this array \\
\hline $\mathrm{P}_{\mathrm{i}}[$ node_id][status] & $\begin{array}{l}\text { Neighbors of node } \mathrm{i} \text { are stored in this array } \\
\text { along with their status. }\end{array}$ \\
\hline next_node & Next node chosen during the traversal \\
\hline $\mathrm{D}$ & $\begin{array}{l}\text { The distance between the last and second } \\
\text { last visited nodes. }\end{array}$ \\
\hline Total & $\begin{array}{l}\text { Total distance traveled during one } \\
\text { simulation }\end{array}$ \\
\hline node_max_pow[] & $\begin{array}{l}\text { The neighboring nodes with the maximum } \\
\text { power are stored in this array }\end{array}$ \\
\hline
\end{tabular}




\subsection{Description}

\subsubsection{Find_Route( )}

Step 1: Begin.

Step 2: Read $\mathrm{n}$ ( $\mathrm{n}$ is total number of nodes) and $\mathrm{p}$ ( $\mathrm{p}$ is initial power for all the nodes)

Step 3: Create the network with $\mathrm{n}$ nodes and set the initial value $\mathrm{p}$ for every node.

Step 4: The source node $m$ sense the event and set the priority value according to event intensity as ep : $2 / 1$ (high/low)

Step 5: If ep $=2$ then do-

a) Find the shortest path from source node $m$ to base station and store all the nodes of this path in the array s_path[ ].

b) $\quad / *$ Reduce the power to transmit the packet from source node $\mathrm{m}$ to base station and find out the remaining average power of the network. */

Step 6: Else Call function Reduce_Power ( )

a) Find the neighboring nodes of source node $(\mathrm{m})$ and store them in the array 'p'.

b) Repeat following steps for $\mathrm{n}$ times ( $\mathrm{n}$ is the neighbor count of source $\mathrm{m}$ )

If status $\mathrm{i}=$ visited then delete $\mathrm{i}^{\text {th }}$ node from Visit[node_id]

c) If all the neighboring nodes are already visited or have the same power value, then choose any one randomly.

Else

Find the node among (not visited) nodes with the maximum power and store in the array 'node_max_power'.

d) If more than one node exists with maximum power content, then select any of them randomly as the next hop and store it as 'next_node'.

e) If the chosen next hop node falls on the base station then-

Else

Call function Reduce_Power ( )

Make the next hop node as the source node $(m)$ and continue the process from step 6(a).

Step 7: End.

\subsubsection{Reduce_Power ( )}

//Reduce the power to transmit the packet from source node $\mathrm{m}$ to base station

Step 1: Begin

Step 2: For $\mathrm{i}=1$ to s_node (which is total number of nodes in s_path[ ]) repeat step (a)

a) For $j=1$ to $n$ repeat the following step

If s_path[i] = power[1][j], then Set power[2][j]=power[2][j]-trans_power

// Find the average power of the network

Step 5: For $\mathrm{i}=1$ to $\mathrm{n}$ repeat the following

Set total $=$ total + power[2][i]

Step 6: Set avg_power=total/n

Step 7: Return

\subsection{Sensor Node Details}

Sensor nodes which are capable of sensing, processing, collecting information and communicating with other nodes participating in the network are the smallest functional units of 
International Journal Of Advanced Smart Sensor Network Systems ( IJASSN ), Vol 2, No.4, October 2012

any wireless sensor network thus the success of a network largely depends on the proper design of sensor nodes. The characteristics and functional specifications of the sensor nodes used in this proposed network are given below.

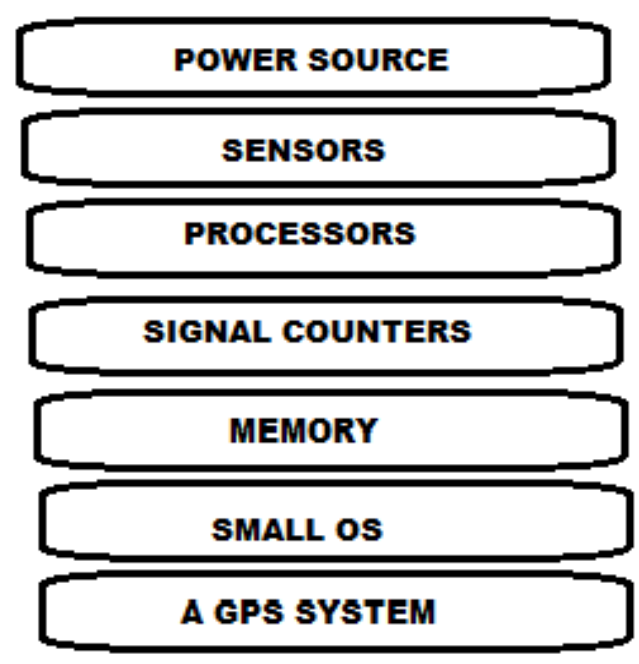

Figure 1. Node components

Each sensor node in the network has same components as depicted in Figure 2 and they are of same type. Every sensor node has various sensors like temperature sensor, pressure sensor, thermo sensor, high density metal sensor s etc. to collect information from the environment of its operating area. Power supply will come from rechargeable and non- rechargeable batteries, processing and decision making work is handled by a high-efficient embedded tiny OS and inbuilt memory. Nodes will also be passive GPS system enabled for the tracking purpose of a node.

\subsection{Case Study}

Let's consider a sensor network of eleven nodes. The values along with nodes are representing their powers and that of edges are representing the distance between the nodes.

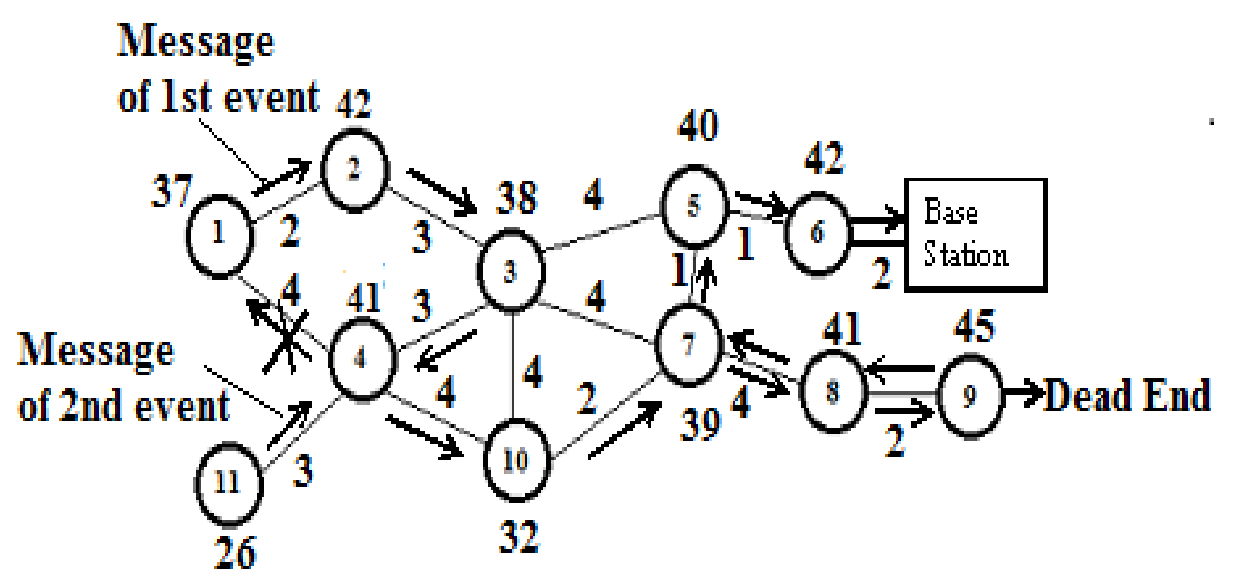

Figure 2. Routing for multiple event by checking infinite loop and dead end 
In the above figure node 1 and node 11 are the source nodes. The first one that is node 1 is sending the data packet of lower priority event when the second one that is node 11 is sending the data packet of higher priority event. At first node 1 will send the packet to node 2 as the power of node 2 (42 in this case) is greater than node 4 ( 41 in this case). Then node 2 will send it to node 3 . Then node 3 will find that node 4 have the maximum power among its neighbours and send the packet to node 4 . Now node 4 will have two packet of different priority one from node 3 (coming from node 1) and another one from node 11 . As packet of node 11 is of higher priority event it will be sent first by shortest path that is node 4-node 3-node 5-node 6-Base Station. After that node 4 will send the packet of lower priority to node 10 as node 1 is already visited, though power of node 1 is greater than node 10 . Then node 10 will send that to node 7 to node 8 to node 9 (according to maximum power), which is a dead end. So the packet will again come back to node 8 (packet header has the node number which are previously visited) and node 7 . The neighbours of node 7 are node 10 , node 3 and node 5 . Node 10 is already visited and node 5 has the maximum power. So the packet will go to node 5 and then to node 6 and at last to the Base station.

\section{Simulation RESUlt}

A network with eleven sensor nodes has been created to analyse the performance of the algorithm. The initial power for every node is considered to 50 units. To simulate the algorithm two high priority and eight low priority messages have been sent from two different source nodes to one base station. The simulation parameters are given in the following table.

Table 2. Parameter list

\begin{tabular}{|l|l|}
\hline \multicolumn{1}{|c|}{ Parameters } & \multicolumn{1}{c|}{ Description } \\
\hline Network size & 11 nodes \\
\hline Initial energy & 50J per node \\
\hline MAC Protocol & IEEE 802.15.4 \\
\hline Power consumption & $\begin{array}{l}\text { Equivalent to packet size and } \\
\text { distance }\end{array}$ \\
\hline Number of message & At least 10 \\
\hline
\end{tabular}

After sending each message the average power of the network has measured and a graph of average power of the network vs. number of messages sent has drawn. It is also compared with the algorithm MERCC [1], where data packets of low priority event are sent without checking for infinite loop or dead end condition. 


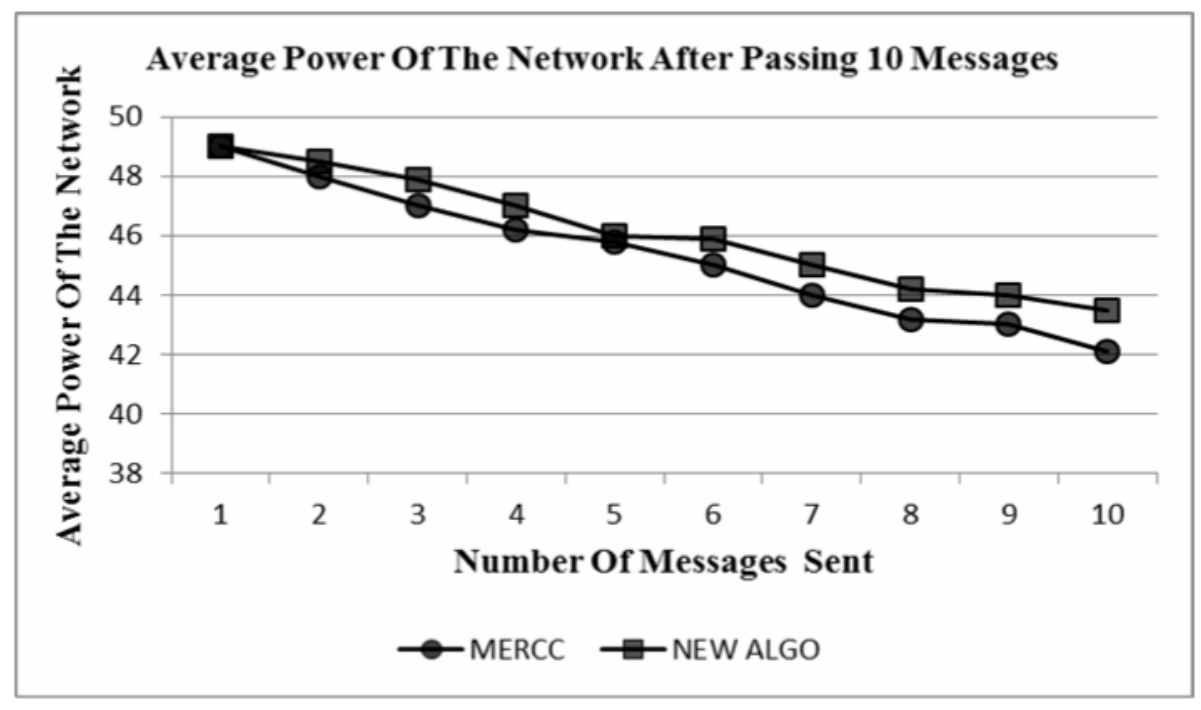

Figure 3. Average power v/s. Number of messages graph

In the above figure message 4 and message 8 are of high priority and thus propagating in shortest path, while the other messages are of low priority and is propagating considering maximum power. It is clear from the above figure that if the messages are sent only considering the maximum power of the neighbour nodes as stated in the algorithm MERCC [1], after passing 10 messages the average power of the network will go down to 42.1 units from 50 units. Where as, if we sent packets according to the proposed algorithm then after passing 10 messages the average power of the network becomes 43.5 units. Therefore the power dissipation of the network according to the proposed algorithm is $14 \%$ less than the algorithm MERCC [1]. Thus this algorithm is optimizing the power consumption of the entire network and also guarantying the delivery of the urgent message in minimum time by controlling the congestion.

\section{Conclusions}

Transmission of urgent information about the devastating event to the base station in a very short span of time is the main objective of wireless sensor network, so that the base station can take corrective action as soon as possible. This should be done with optimized power consumption for the shake of longevity of the network. The current state of the art study shows that most of the power saving algorithms fails to take care of timeliness of delivery and the probability of multiple event occurrences at the same instance. The proposed algorithm states an event-driven routing methodology to handle multiple events at a single instance with minimum energy drainage. This method is also concerned about the infinite loop problem, data loss and takes care of the congestion within the network. The simulation result shows the proposed algorithm increases the lifetime of the network by 14\%, as compared with our earlier algorithm MERCC [1].

\section{REFERENCES}

[1] Ayan Kumar Das, Dr. Rituparna Chaki, "MERCC: Multiple Events Routing with Congestion Control for WSN" in Proceedings of The Second International Conference on Advances in Computing and Information Technology(ACITY 2012), Chennai, India, Advances in Intelligent Systems and Computing, 1, Volume 176, Advances in Computing and Information Technology, Pages 691-698, 2012. 
[2] Karim,L., Nasser,N.; ElSalti,T.; Sch. of Comput. Sci., Univ. of Guelph, Guelph, ON, Canada, "Efficient Zone-based Routing Protocol of Sensor Network in agriculture monitoring systems", Communications and Information Technology (ICCIT), ISBN: 978-1-4577-0401-7, Issue Date: 29-31 March 2011.

[3] Ayan Kumar Das, Dr. Rituparna Chaki, "A Novel Power Balancing Routing Scheme for WSN" in Proceedings of The Third International Conference on Wireless \& Mobile Networks (WiMo-2011), Ankara, Turkey will be published by Springer (LNCS) in Communications in Computer and Information Science (CCIS) Series,Issue-June,2011.

[4] Ayan Kumar Das, Debaditya Ghosh, Pritam Majumder, "Intelligent Energy Efficient Routing For Ad Hoc Sensor Network by Designing QCS Protocol" in Proceedings of The Second International Workshop on Adhoc, Sensor and Ubiquitous Computing (ASUC-2011) will be published by Springer (LNCS) in Communications in Computer and Information Science (CCIS) Series,Issue-June,2011.

[5] Sourabh Jain, Praveen Kaushik and Jyoti Singhai, "Greedy Heuristic Based Energy Efficient Routing in Wireless Sensor Network", in proceedings of Communications in Computer and Information Science, 2011, Volume 203, Part 1, 282-292, DOI: 10.1007/978-3-642-24037-9_27.

[6] GONG Ben-can LI La-yuan JIANG Ting-yao XU Shou-zhi, "Distributed Spanning Tree-Based Routing Protocol for Wireless Sensor Networks", MICROELECTRONICS \& COMPUTER, 2008, 25(11).

[7] Siva Kumar. D and Bhuvaneswaran.R.S, "Proposal on Multi agent Ants based Routing Algorithm for Mobile Adhoc Networks", in IJCSNS International Journal of Computer Science and Network Security, Vol-7, No. 6, June,2007.

[8] Tiago Camilo, Carlos Carreto, Jorge Sá Silva and Fernando Boavida, “An Energy-Efficient AntBased Routing Algorithm for Wireless Sensor Networks", Lecture Notes in Computer Science, Volume 4150/2006, 49-59, DOI: 10.1007/11839088_5, 2006.

[9] V.Laxmi, Lavina Jain, M.S.Gaur, "Ant Colony Optimization based Routing on NS-2" in the proceedings of International Conference On Wireless Communication and Sensor Networks, WCSN 2006.

[10] Shih-Chang Huang; Rong-Hong Jan; Dept. of Comput. \& Inf. Sci., Nat. Chiao Tung Univ., Hsinchu, Taiwan, "Energy-aware, load balanced routing schemes for sensor networks", ISSN: 1521-9097, Issue Date: 7-9 July 2004.

[11] J.QiangFeng and D.Manivannan, "Routing protocols for sensor networks", in Consumer Communications and Networking Conference, CCNC 2004.First IEEE.2004.

[12] W.R.Heinzelman, A.Chandrakasan, and H.Balakrishnan, "Energy Efficient Communication Protocol for Wireless Microsensor Networks", In Proceedings of the 33rd Hawaii International Conference on System Sciences, 2000.

[13] Godfrey P. and Ratajczak D. Naps: Scalable, "Robust topology management in wireless ad hoc networks". Proceedings of the third International Symposium on Information Processing in Sensor Networks, 2004

[14] Younis,O.; Fahmy, S.; Dept. of Comput. Sci., Purdue Univ., West Lafayette, IN, USA, "HEED: a hybrid, energy-efficient, distributed clustering approach for ad hoc sensor networks", appears in Mobile Computing IEEE Transactions on Volume: 3 Issue: 4, ISSN: 1536-1233 Issue Date: Oct.-Dec. 2004.

[15] M. Gunes, U. Sorges, I. Bouazizi, “ARA- the ant colony based routing algorithm for MANET”,ICPP Proc of the 2002.

[16] Handy, M. Haase, M. Timmermann, D., "Low Energy Adaptive Clustering Hierarchy with Deterministic Cluster Head Selection", 4th IEEE International Conference on Mobile and Wireless Communication Networks, Stockholm, 2002.

[17] S. Slijepcevic and M. Potkonjak, "Power efficient organization of wireless sensor networks", in IEEE International Conference on Communications", Helsinki, Finland, June 2001.

[18] W.R.Heinzelman, A.Chandrakasan, and H.Balakrishnan, "Energy Efficient Communication Protocol for Wireless Microsensor Networks", In Proceedings of the 33rd Hawaii International Conference on System Sciences, 2000.

[19] Intanagonwiwat, C. Govindan R. and Estrin, D. Directed Diffusion: A Scalable and Robust Communication Paradigm for Sensor Networks. In Proceedings of the sixth Annual International Conference on Mobile Computing and Networks (MobiCOM2000), 2000. 


\section{Authors}

1. Ayan Kumar Das is an Assistant Professor in the Department of Information Technology, Calcutta Institute of Engineering and Management, Kolkata, India, since 2005. He has passed M.Tech in Software Engineering from West Bengal University of Technology, India. Presently he is a PhD scholar in the department of Computer Science and Engineering, University of Calcutta, India. The primary area of research interest for Mr. Das is Wireless Sensor Network.

2. Rituparna Chaki is an Associate Professor in the Department of Computer Science \& Engineering, West Bengal University of Technology, Kolkata, India, since 2005. She received her $\mathrm{Ph}$. D. in 2002 from Jadavpur University, India. The primary area of research interest for Dr. Chaki is Wireless Mobile Ad hoc Networks and Data Mining. She has also served as a Systems Manager for Joint Plant Committee, Government of India for several years before she switched to Academia. Dr. Chaki also serves as a visiting faculty member in other leading Universities including Jadavpur University. Dr. Chaki has about 45 referred international publications to her credit.
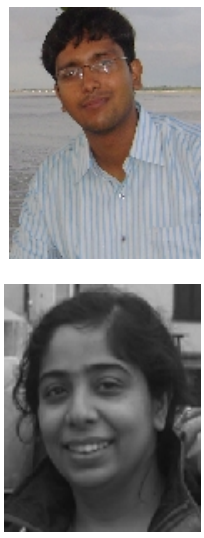